\title{
El valor de la evaluación de las relaciones públicas y de la comunicación en las organizaciones: el caso de los hospitales del Sistema Nacional de Salud Español
}

\author{
Guillerm Marca ${ }^{1}$ \\ Andrea Oliveira² \\ Kathy Matilla ${ }^{3}$ \\ Thais Miranda ${ }^{4}$
}

Recibido: 2015-10-27

Enviado a pares: 2016-01-17
Aprobado por pares: 2016-05-18

Aceptado: 2016-06-02

DOI: 10.5294/pacla.2017.20.2.9

Para citar este artículo / to reference this article / para citar este artigo

Marca, G., Oliveira, A., Matilla, K. y Miranda, T. (2017). El valor de la evaluación de las relaciones públicas y de la comunicación en las organizaciones: el caso de los hospitales del Sistema Nacional de Salud Español. Palabra Clave, 20(2), 506-528. DOI: 10.5294/ pacla.2017.20.2.9

\section{Resumen}

Este artículo pretende determinar si los hospitales del Sistema de Salud Español desarrollan actividades de evaluación de sus campañas de relaciones públicas y comunicación y establecer cuál es su propósito. Como objetivos secundarios, se indagó sobre el presupuesto destinado a dicha evaluación y sobre si las características del proceso de evaluación de los hospitales se adaptaban a los postulados de Besson. Se tomó una amplia muestra de 300

\footnotetext{
1 orcid.org/0000-0002-1586-8625. Universitat de Vic - Universitat Central de Catalunya (UVic-UCC). España. guillem.marca@uvic.cat

2 orcid.org/0000-0002-1370-6139. Universitat de Girona. España. andrea.oliveira@udg.edu

3 orcid.org/0000-0001-5247-3289. Blanquerna - Universitat Ramon Llull. España. kathyms@blanquerna.url.edu

4 orcid.org/0000-0001-8315-0562. Universidade Federal da Bahia. Brasil. thaisbmiranda@gmail.com
} 
hospitales públicos españoles (tasa de respuesta de $23.33 \%$ ). Los resultados indican que los hospitales llevan a cabo evaluaciones para el desarrollo de sus funciones, pero no destinan grandes partidas presupuestarias para evaluar sus acciones. Ello sugiere que existe una preocupación por demostrar el valor de la práctica de las relaciones públicas en los hospitales, pero que aún se encuentran en una fase inicial de adopción sistemática de una forma concreta de modelo del uso estratégico de la evaluación.

\section{Palabras clave}

Evaluación; valor; hospital; relaciones públicas; comunicación; eficacia; Sistema Nacional de Salud (Fuente: Tesauro de la Unesco). 


\section{The Value of the Evaluation of Public Relations and Communication in Organizations: The Case of the Spanish National Health System Hospitals}

\section{Abstract}

This article attempts to determine whether the hospitals of the Spanish Health System carry out evaluation activities of their public relations and communication campaigns and to establish their purpose. As secondary objectives, the budget for the evaluation was examined, as well as if the characteristics of the hospital evaluation process were adapted to Besson's postulates. A large sample of 300 Spanish public hospitals (response rate of $23.33 \%$ ) was taken. The results indicate that hospitals carry out evaluations for the development of their duties, but that they do not allocate a large budget to evaluate their actions. This suggests that there is a concern to demonstrate the value of public relations practice in hospitals but that they are still at an early stage of a systematic adoption of a concrete form of model for the strategic use of evaluation.

\section{Keywords}

Evaluation; value; hospital; public relations; communication; effectiveness; National Health System (Source: Unesco Thesaurus). 


\section{0 valor da avaliação das relações públicas e da comunicação nas organizações: 0 caso dos hospitais do Sistema Nacional de Saúde Espanhol}

\section{Resumo}

Este artigo pretende determinar se os hospitais do Sistema de Saúde Espanhol desenvolvem atividades de avaliação das suas campanhas de relações públicas e comunicação e estabelecer qual é seu propósito. Como objetivos secundários, se indagou sobre o orçamento destinado a esta avaliação e sobre se as caraterísticas do processo de avaliação dos hospitais se adaptavam aos postulados de Besson. Tomou-se uma ampla amostra de 300 hospitais públicos espanhóis (taxa de resposta de $23.33 \%$ ). Os resultados indicam que os hospitais levam a cabo avaliações para o desenvolvimento de suas funções, mas não destinam grandes partidas orçamentárias para avaliar suas ações. Isso sugere que existe uma preocupação por demonstrar o valor da prática das relações públicas nos hospitais, mas que ainda se encontram em uma fase inicial de adoção sistemática de uma forma concreta de modelo do uso estratégico da avaliação.

\section{Palavras-chave}

Avaliação; valor; hospital; relações públicas; comunicação; eficácia; Sistema Nacional de Saúde (Fonte: Tesauro da Unesco). 


\section{Introducción}

Demostrar la eficiencia de las campañas de relaciones públicas y comunicación desarrolladas es imprescindible en las organizaciones. En este sentido, en los últimos años del siglo $\mathrm{xx}$, los académicos de las relaciones públicas y la comunicación se mostraron activos en el debate y en el desarrollo de metodologías y programas de formación de los profesionales en la evaluación de la disciplina. Como respuesta, algunos profesionales comenzaron a evaluar sus actividades en las organizaciones (Watson, 2012). Las iniciativas de mejora de la calidad y la cantidad de evaluación de los programas se deben, por un lado, a la necesidad de los profesionales de relaciones públicas y comunicación de demostrar su contribución al funcionamiento eficaz de las organizaciones y, por otro, a que la alta dirección está exigiendo conocer los resultados y el retorno a la inversión de sus asignaciones presupuestarias (Gregory, 2001).

La evaluación de los programas de relaciones públicas y comunicación desempeña un papel importante en la rendición de cuentas y en la demostración de la eficacia (Dozier, 1990; Fairchild, 2002; Xavier et al., 2005), así como en el impacto en la estrategia corporativa y de negocio de toda organización (Radford y Goldstein, 2002, Xavier et al., 2005). A estos elementos, Theilmann y Szondi (2009) añaden que la evaluación es el garante de la profesionalidad del programa, y establece con ello una dimensión de verificación de la reputación de la disciplina y de la práctica profesional, añadiendo que es transversal en la práctica diaria y que es un punto de interés común, compartido entre profesionales y académicos.

Para los profesionales, la rendición de cuentas se dirige a los clientes, pero también es de utilidad para con ellos mismos (Bissland, 1990), ya que solo de esta forma conseguirán posicionarse adecuadamente ante las "coaliciones dominantes" a las que se refieren las definiciones de la profesión que le dan el rango de función directiva. Las direcciones funcionales requieren un conjunto de conocimientos basados en la investigación y en las técnicas de la evaluación para proporcionarles legitimidad y valoración empírica, más allá de la mera opinión subjetiva (Watts, 2006). El caso de la gestión de las relaciones públicas y la comunicación no es diferen- 
te (Jefferey y Burton, 2012). Como establece Gregory (1996), si se consigue el logro de los objetivos, nadie puede dudar del valor del profesional, ya que el adecuado uso de la evaluación demuestra la efectividad y la eficacia de la inversión realizada como elementos prioritarios para alcanzar resultados excelentes. La evaluación, pues, legitima la priorización de actividades primordiales respecto de otras de carácter secundario, tanto en el departamento como en toda la organización.

En un estudio Delphi desarrollado por Watson (2008), se observó que una de las prioridades principales de la investigación de evaluación de las relaciones públicas fue el papel de estas para contribuir al desarrollo de la toma de decisiones y el funcionamiento estratégico de la organización. No obstante, a pesar de los debates académicos y profesionales y las teorías desarrolladas y aceptadas sobre la medición y la evaluación, los profesionales se han mostrado reacios a adoptar métodos de eficacia probada en sus prácticas diarias (Watson, 2012). En esa misma línea, otros estudios subrayan que los profesionales de las relaciones públicas y la comunicación hablan con frecuencia acerca de la importancia de la evaluación, pero no la llevan a la práctica de forma prevalente (Watson, 1994; Wright et al., 2009), y aumenta la pérdida de credibilidad de las relaciones públicas y del valor de su evaluación (Noble, 1999).

Si bien la mayoría de los estudios sobre la evaluación en la práctica de las relaciones públicas y la comunicación llevados a cabo en las últimas dos décadas aportan abundantes afirmaciones de reconocimiento positivo hacia la evaluación de programas (Lindenman, 1993; Macnamara, 1999, 2002; Walker, 1997; Watson y Noble, 2007), son menos los que analizan si estas se llevan a cabo. Destacan los resultados de los estudios de Watson (1994,1997), realizados también en colaboración con otros colegas, en los que $75 \%$ de los profesionales participantes en 1992 y $80 \%$ en 1996 afirmaba llevar a cabo evaluaciones de sus programas (Watson y Noble, 2007). Simons y Watson (2005) replicaron la encuesta en Australia, y observaron una respuesta afirmativa de $80 \%$. No obstante, una década después, en un estudio similar, Xavier, Mehta y Gregory (2006) obtuvieron una respuesta afirmativa de solo $50 \%$. 
Un $52.8 \%$ de los encuestados por Lindenmann en 1990 afirmó destinar entre 1 y $6 \%$ a la evaluación. Watson, en su estudio de 1992, estableció que $74.3 \%$ de los encuestados destinaba entre 0 y $5 \%$ de su presupuesto a la evaluación (Watson y Noble, 2007).

Con la Barcelona Declaration of Measurement Principles (AMEC, 2010), académicos y profesionales pretendieron dar un nuevo impulso a la evaluación de las relaciones públicas y la comunicación y contribuir así a valorar la disciplina y a facilitar $y$ alentar a los profesionales a realizar un adecuado uso de la evaluación.

El presente estudio se centró en uno de los sectores en los que la investigación y la comprobación de resultados forman parte de su ADN cultural, el sector de la salud, concretamente el ámbito hospitalario. Como objetivo prioritario, se investigó si los departamentos de relaciones públicas y comunicación del Sistema Nacional de Salud utilizaban la evaluación y el presupuesto destinado a dicho procedimiento y si abordaban sus procesos de evaluación a partir de los principios establecidos por Besson (2008), cuyo modelo consta de tres divisiones: 1) las condiciones previas para la evaluación de las relaciones públicas, 2) la evaluación del programa y 3) la explotación de los resultados. El modelo se construye en forma de un círculo, ya que esta forma apoya el requisito de continuidad. Al mismo tiempo, existe una función continua de retroalimentación que acompaña a todas las fases. Esta función implica la existencia de un coordinador, que revisa y mantiene el plan de evaluación y pone los resultados de esta a disposición del director de relaciones públicas durante el proceso. De esta manera, es posible optimizar el programa de relaciones públicas inmediatamente.

\section{Evaluación de la comunicación en salud}

Si bien existe amplia literatura sobre comunicación y salud (Busto, 2016; Medina, 2012; Wise, 2009, 2012), dirigida a nuestro objeto de estudio, la evaluación de la comunicación, al ámbito de la salud, y concretamente al ámbito de la comunicación en el hospital, son muy pocos los autores que abordan el tema y lo hacen de forma superficial y vaga, totalmente alejada de los parámetros establecidos por AMEC (2010). Defienden la creación de un departamen- 
to de comunicación motivado por las necesidades comunicativas internas y externas del hospital, y le atribuyen el diseño, la planificación, la ejecución, la evaluación y la coordinación de la estrategia comunicativa - hacia adentro y hacia fuera-, sin profundizar en la evaluación (Costa, 2011), o reducen la evaluación a una "variable de proceso" (Díaz y Cuesta, 2014) y omiten la importancia aportada por la Declaración de Barcelona.

Desde un análisis de los roles de los profesionales de relaciones públicas de los departamentos de comunicación de los hospitales se identifica la investigación evaluativa como una de las actividades que menos se lleva a cabo, pero no se profundiza en ello (Bosilkovski y Lee, 2013). No obstante, se reconoce el interés de distintos organismos de acreditación estadounidenses de evaluaciones independientes de la satisfacción del paciente. La medición continua puede ayudar a medir el éxito de las mejoras de servicios, el impacto de las nuevas ofertas de servicios y los resultados de marketing/esfuerzos de promoción (Hogan, Hershey y Ritchey, 2008).

A partir del marco científico revisado, las preguntas de investigación que se plantearon fueron:

PI1: ¿Los departamentos de relaciones públicas y comunicación del Sistema Nacional de Salud llevan a cabo evaluaciones de sus programas?

PI2:¿Qué presupuesto destinan a dicho procedimiento?

PI3: ¿Con qué propósito realizan las evaluaciones?

PI4: ¿Están vinculadas las evaluaciones a unos objetivos preestablecidos?

PI5: ¿Cuál es el uso que se da a los resultados de las evaluaciones?

\section{Metodología}

\section{Desarrollo de la encuesta}

Para la investigación, se utilizó un cuestionario autoadministrado. La encuesta se desarrolló mediante un trabajo de síntesis de encuestas previas 
centradas en la evaluación de las relaciones públicas y la comunicación, tanto en el ámbito internacional, a partir de los estudios realizados por Dozier (1981, 1984), Macnamara (1999), Lindenmann (1990), Watson (1994), Walker (1997), Watson y Simmons (2004) y Xavier (2006), como en el ámbito nacional, según los estudios llevados a cabo por Huertas (2009) y Armendáriz (2010). Posteriormente, se consultó, para su validación, con un grupo de 25 expertos españoles en el campo de las relaciones públicas, la comunicación y la gestión hospitalaria, mediante entrevistas en profundidad (Kvale, 1996)a 10 académicos y a 15 profesionales en activo.

\section{Selección de la muestra}

La procedencia de la población objeto de estudio fueron todos los centros hospitalarios que formaban parte del Sistema Nacional de Salud Español. Se obtuvo una base de datos de centros hospitalarios públicos a partir del Ministerio de Sanidad y Consumo, Catálogo Nacional de Hospitales (2010).

\section{Criterios de inclusión y de exclusión}

Para la selección de la muestra se incluyeron hospitales públicos de más de 200 camas y que estas fueran destinadas a la asistencia de patología aguda. Se excluyeron aquellos centros cuya asistencia fuera, de forma monográfica, la rehabilitación, la enfermedad psiquiátrica, la geriatría y la larga estancia. Tras llevar a cabo dicha depuración, la muestra definitiva quedó configurada por 300 hospitales.

\section{Administración de la encuesta}

El cuestionario se envió, mediante enlace en un correo electrónico, a todos los gerentes de los 300 hospitales de la muestra definitiva. Estos, generalmente, transmitían el cuestionario al director de relaciones públicas o comunicación -o equivalente - para que lo respondieran. Se efectuaron tres envíos consecutivos, entre el 15 de mayo y el 15 de junio de 2014, a 300 direcciones de correo electrónico y se obtuvieron 70 respuestas (tasa de respuesta de $23.33 \%$ ).

\section{Contenido del cuestionario}

Los encuestados fueron preguntados por aspectos relacionados con la integración de la evaluación como una función directiva en el ámbito de la co- 
municación, ya sea en forma de valorar su importancia en cualquier caso, ya sea sobre el presupuesto destinado a esta función. Además, debían aceptar o negar afirmaciones que definían diferentes formas de redacción de los objetivos de un programa de relaciones públicas y comunicación y sobre los usos y fines de la investigación de evaluación.

\section{Análisis estadístico}

Las principales medidas resultado fueron estas: afirmación sobre llevar a cabo evaluaciones, porcentaje del presupuesto del departamento destinado a evaluaciones, motivos por los que se lleva a cabo la evaluación, características de los objetivos del plan de comunicación, momento en el que se plantea la evaluación y el uso de la redacción de informes de evaluación, sus métodos y su frecuencia de difusión. Para describir variables categóricas, se utilizó el número total de respuestas $(N)$ y el porcentaje de cada categoría y utilizamos una prueba de chi-cuadrado de tendencia lineal para el análisis bivariado. Un valor $P$ de menos de 0.05 se consideró estadísticamente significativo. El nivel de significación tenido en cuenta para todos los análisis de este estudio ha sido de $95 \%$. La información obtenida fue procesada electrónicamente mediante el paquete estadístico de software Statistical Packageforthe Social Sciences (SPSS) versión 14, para su ordenamiento y análisis.

\section{Resultados}

Un total de 70 responsables de los departamentos de relaciones públicas y comunicación de los 300 hospitales de la muestra respondieron de forma válida las encuestas (tasa definitiva de respuesta de $23.33 \%$ ), en su mayoría $(74.3 \%, \mathrm{n}=52)$ hospitales públicos y de más de 400 camas $(80 \%, \mathrm{n}=56)$.

PI1: ¿Los departamentos de relaciones públicas y comunicación del Sistema Nacional de Salud llevan a cabo evaluaciones de sus programas?

Los participantes de la muestra de los hospitales del Sistema Nacional de Salud afirmaron llevar a cabo evaluaciones $(75.7 \%, \mathrm{n}=53)$. Del resto, $18.6 \%(\mathrm{n}=13)$ declaró no evaluar y $5.7 \%(\mathrm{n}=4)$ respondió "no saber". 


\section{PI2: ¿Qué presupuesto destinan a dicho procedimiento?}

Cuando preguntamos a los responsables de relaciones públicas y comunicación si en el presupuesto de su centro hospitalario se incluía una partida de fondos destinados a la investigación o a la evaluación de las relaciones públicas y la comunicación, $20 \%(\mathrm{n}=14)$ respondió afirmativamente. En contrapartida, $68.6 \%(\mathrm{n}=48)$ reconoció no incluir esta partida.

En cuanto al porcentaje del presupuesto del departamento de relaciones públicas y comunicación destinado a la investigación o a la evaluación, $41.4 \%(\mathrm{n}=29)$ de los encuestados afirmó dedicar menos de $1 \% ; 27.1 \%$ $(\mathrm{n}=19)$ dedicó entre 1 y $5 \%$; y 14 encuestados $(20 \%)$ destinaron entre 6 y $10 \%$. Ocho responsables de departamento $(11.42 \%)$ no destinaron ninguna partida del presupuesto del departamento a la evaluación de sus tareas.

\section{PI3: ¿Con qué propósito realizan las evaluaciones?}

Los motivos por los que se lleva a cabo la evaluación en los departamentos de relaciones públicas y comunicación de los hospitales son diversos. El más destacado de todos es el de formar a los compañeros sobre las actitudes y las opiniones de los grupos de interés, variable en la que $88.6 \%$ $(\mathrm{n}=62)$ estaba de acuerdo o muy de acuerdo. En segundo lugar, los encuestados afirmaron defender el departamento ante el resto de departamentos del hospital, $61.4 \%(n=43)$. Les siguen, en sentido descendente, el uso de la evaluación para justificar el presupuesto del departamento $(50 \%, \mathrm{n}=35)$, formar a la alta dirección sobre el valor de las relaciones públicas y la comunicación para el hospital $(48.6 \%, \mathrm{n}=34)$; y, por último, evaluar con propósitos de planificación estratégica $(31.4 \%, \mathrm{n}=22)$ (figura 1$)$.

PI4: ¿Están vinculadas las evaluaciones a unos objetivos preestablecidos?

La planificación de la evaluación se planteó, en la mayoría de los casos, al final de los programas de comunicación $(81.4 \%, \mathrm{n}=57)$. Los encuestados afirmaron que, cuando planteaban los objetivos, no tenían en cuenta su futura forma de evaluación $(88.6 \%, \mathrm{n}=62)$. 


\section{Figura 1. Usos y propósitos de la evaluación}

Uso de la evaluación con propósitos de planificación estratégica.

Uso de la evaluación para educar a la alta dirección sobre el valor de la comunicación.

Uso de la evaluación para justificar el presupuesto.

Uso de la evaluación para defender al departamento frente a otros.

Uso de la evaluación para educar a los compañeros de trabajo sobre las actitudes/opiniones de los grupos de...

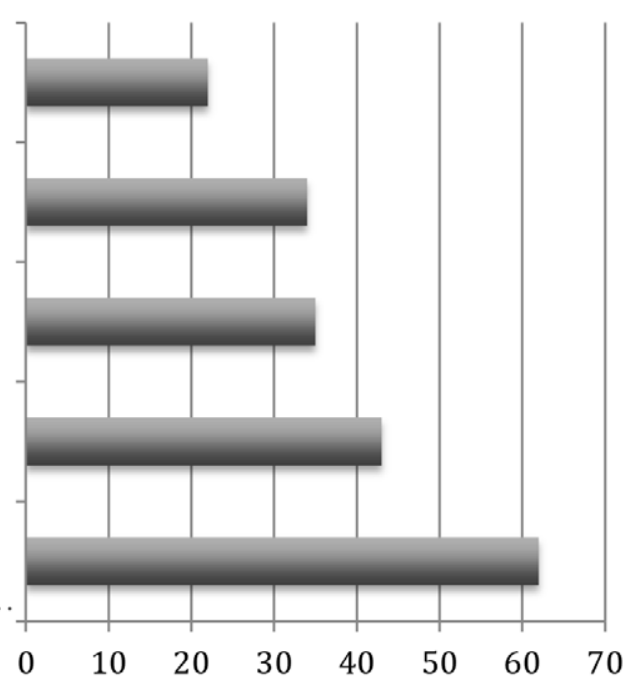

Sobre la forma en que los responsables de los departamentos de relaciones públicas y comunicación abordaban el establecimiento de los objetivos, $54.3 \%(\mathrm{n}=38)$ reconoció redactarlos de forma genérica; $77.1 \%$ $(\mathrm{n}=54)$ los redactó definiendo específicamente el tipo de resultados que se pretendían conseguir con el programa de relaciones públicas y comunicación; $32.9 \%(n=23)$ los redactaba definiendo el porcentaje de público que se pretendía alcanzar; y $63.4 \%(\mathrm{n}=45)$ estableció plazos temporales de implementación y cumplimiento (figura 2).

PI5: ¿Cuál es el uso que se da a los resultados de las evaluaciones?

Todos los encuestados coincidieron en el uso de los resultados de la evaluación para la creación de informes y memorias. Un $81.4 \%(\mathrm{n}=57)$ coincidió en que los informes de resultados son útiles para futuras planificaciones, $92.9 \%(\mathrm{n}=65)$ coincidió en que los informes de resultados intermedios los utilizaban para reajustar el programa, y los informes de resultados finales para redefinir programas futuros $(62.9 \%, \mathrm{n}=44)$. Un $87.3 \%$ $(\mathrm{n}=61)$ estaba de acuerdo en que la creación de estos informes era debida a intereses del propio departamento y afirmaron, en $61.4 \%(n=43)$ de los casos, que su uso ayudaba a redefinir las acciones de relaciones públicas y comunicación (figura 3 ). 


\section{Figura 2. Redacción de objetivos}

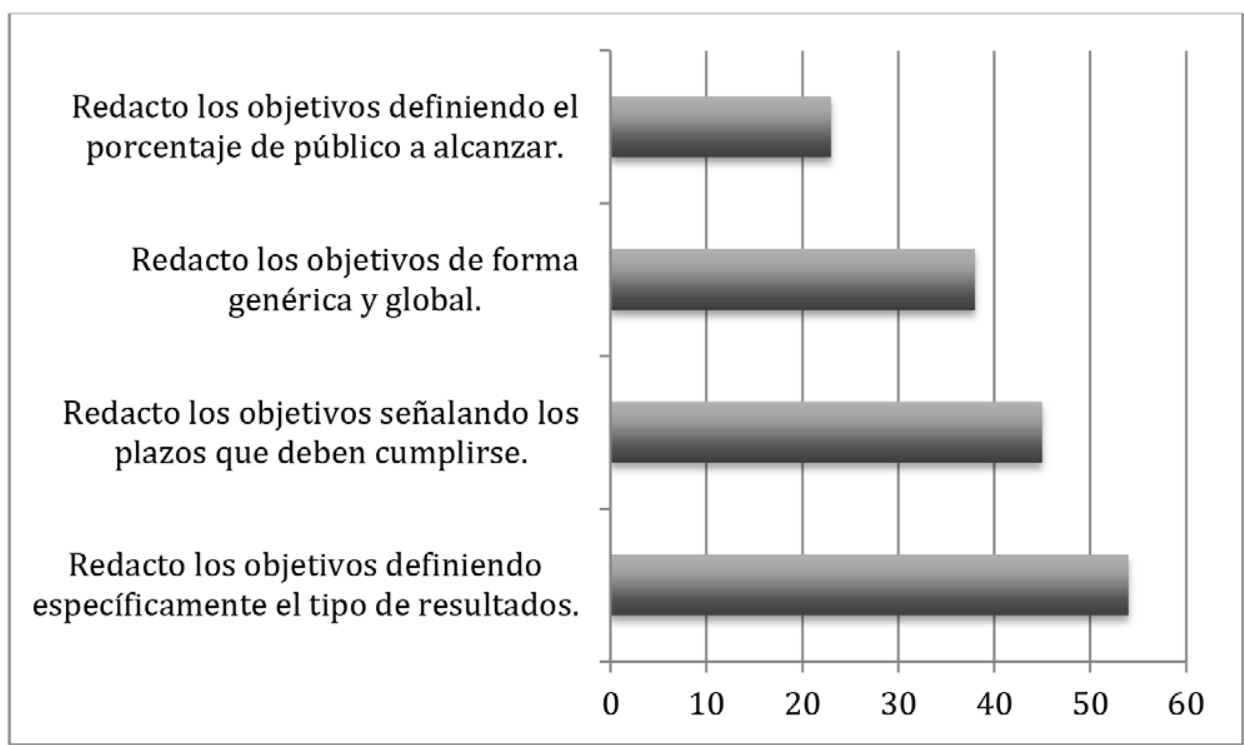

\section{Figura 3. Uso de los resultados}

Utilizar los resultados para redefinir acciones de comunicación.

Utilizar los resultados para intereses del propio departamento.

Utilizar los resultados finales para redefinir futuros programas.

Utilizar los resultados intermedios para reajustar el programa.

Utilizar los resultados para planificaciones futuras.

Utilizar los resultados para la elaboración de informes y memorias.

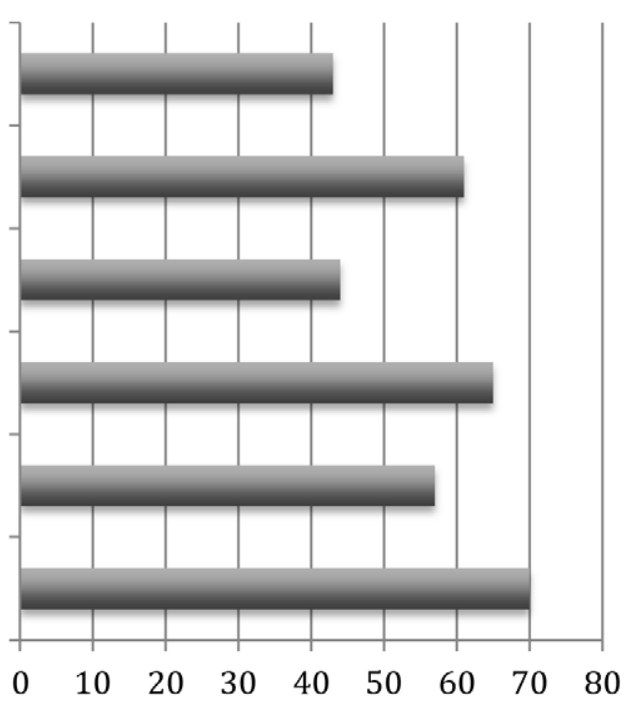


Cuando el destinatario de los informes de la evaluación era la alta dirección, se le informaba, mayoritariamente, en reuniones $(50 \%, \mathrm{n}=35)$ y mediante informes $(37.1 \%, \mathrm{n}=26)$, mientras que, cuando eran los demás departamentos del hospital, o no se les informaba en $30 \%(n=21)$ de los casos o se hacía anualmente $(51.4 \%, \mathrm{n}=36)$. Cuando se les informaba era en reuniones $(25.7 \%, \mathrm{n}=18)$ o a través de la memoria anual $(22.9 \%$, $\mathrm{n}=16$ ). Al colectivo de empleados del centro hospitalario mayoritariamente no se les informaba $(42.9 \%, \mathrm{n}=30)$ o se les informaba anualmente $(38.6 \%, \mathrm{n}=27)$, en reuniones $(20 \%, \mathrm{n}=14)$ o mediante la memoria anual $(17.1 \%, \mathrm{n}=12)$.

Los informes sobre las evaluaciones se transmitían a la opinión pública en muy pocos casos y cuando se hacía era con periodicidad anual (12.9\%, $n=9)$, por medio de la memoria anual $(5.7 \%, n=4)$ (figura 4$)$.

\section{Figura 4. Formas como se informa de los resultados}

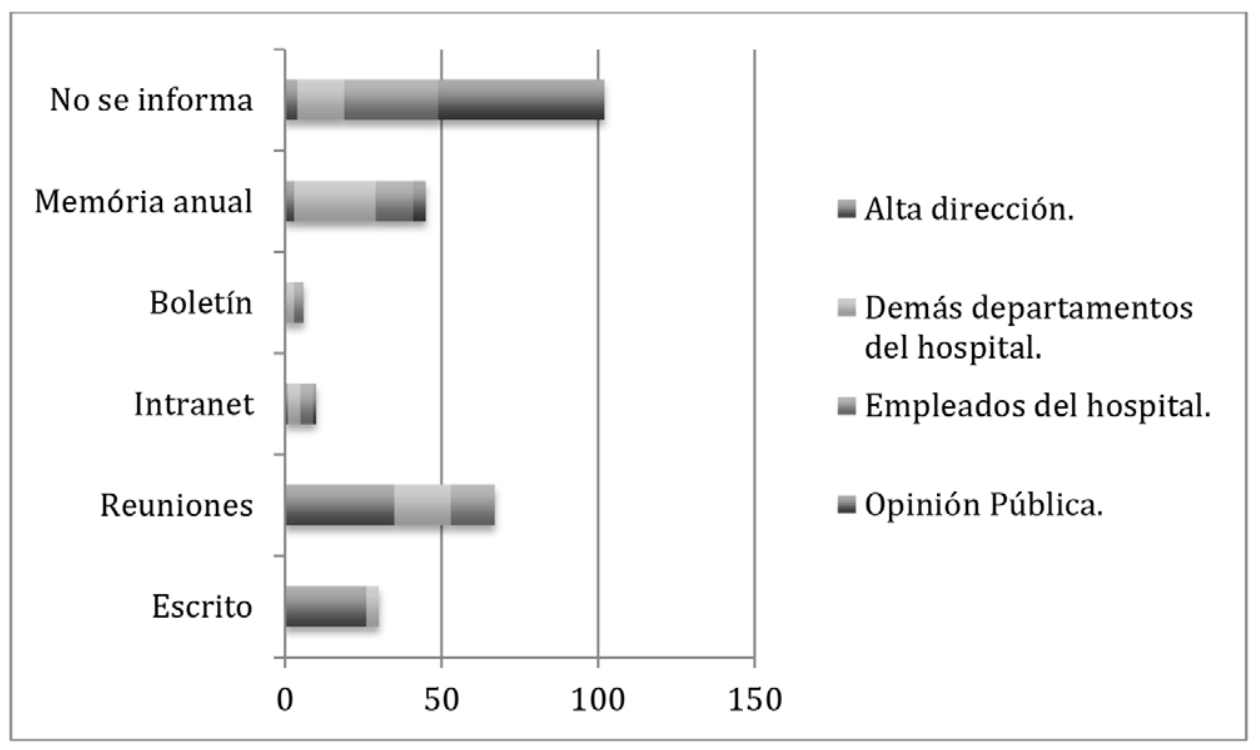

\section{Discusión y conclusiones}

La mayoría de los departamentos de relaciones públicas y comunicación de los hospitales de la muestra afirmaron realizar evaluaciones $(75.7 \%, \mathrm{n}=53)$ de sus programas, en congruencia con Watson y Noble (2007) y Watson y 
Simmons (2004), y 25 puntos porcentuales por encima de los resultados de la investigación de Xavier, Mehta y Gregory (2006). Solo $11.4 \%$ ( $\mathrm{n}=8)$ no destinó partida económica alguna a la evaluación, y los encuestados que destinaban partidas superiores a $1 \%$ del presupuesto del departamento fueron $47.1 \%$ de la muestra. Con ambos datos, se observa que los responsables de los departamentos de relaciones públicas y comunicación de los hospitales participantes evaluaron sus acciones de comunicación, pese a que la mayoría $(68.6 \%, \mathrm{n}=48)$ de los presupuestos de los centros hospitalarios públicos españoles no contemplaron la asignación de partida presupuestaria específica para este fin.

En relación con el presupuesto asignado a la evaluación final de los departamentos de relaciones públicas y comunicación de los hospitales públicos, los resultados hallados en nuestro estudio difieren de los resultados consignados en la literatura especializada citada. El porcentaje del presupuesto del departamento de relaciones públicas y comunicación de los hospitales públicos españoles participantes destinado a la investigación o a la evaluación, en $41.4 \%(\mathrm{n}=29)$ de los casos se dedicó menos de $1 \%$; $27.1 \%(\mathrm{n}=19)$ dedicó entre 1 y $5 \%$ (porcentaje muy inferior a $52.8 \%$ consignado por Lindemann [1990]); y 14 encuestados (20\%) destinaron entre 6 y $10 \%$, porcentajes muy superiores al máximo de $6 \%$ identificado por Lindemann (1990) y de $5 \%$ observado por Watson y Noble (2007). Recordemos que 8 (11.42\%) responsables de departamento participantes declararon no destinar ninguna partida del presupuesto a la evaluación de sus tareas, en consistencia con Watson (2012).

Es destacable que la finalidad por la que los participantes de nuestro estudio lleven a cabo evaluaciones sea la de formar al equipo sobre las actitudes y las opiniones de los grupos de interés. Las dos tipologías de resultados más compartidas (Lindenman, 2002; Paine, 2007; Stacks 2006) están, en el caso de los hospitales participantes, muy por delante de acciones de defensa del departamento o de justificación del presupuesto, uno de los elementos que definen la evaluación desde su dimensión comercial (Watson, 1997). Solo la mitad de los departamentos utilizan los resultados 
de las evaluaciones para demostrar el valor añadido de la profesión ante los altos cargos de la institución hospitalaria.

Demostrar la contribución de las acciones de relaciones públicas y comunicación contribuye al refuerzo de la concepción de la función directiva de la profesión anhelada desde la definición de relaciones públicas de La Haya de 1960 (Wilcox, Cameron y Reber (1992), repetida numerosas veces en la literatura y, según nuestros resultados, todavía no consolidada.

En la misma línea, es destacable el último lugar en el que queda situada la planificación estratégica, lo cual da a entender que, bien la profesión no conoce la disciplina estratégica basada en la investigación y la evaluación, bien no la aplica, tal y como establecieron Matilla y Marca (2012). Estos dos últimos datos se distancian de los postulados de Grunig (2013) cuando afirma: "Las relaciones públicas asumen cada vez más posiciones en cuanto a la estrategia y a la toma de decisiones de la organización, formando coaliciones dominantes".

La Barcelona Declaration of Measurement Principles (AMEC, 2010) destaca la importancia del establecimiento de metas y objetivos para la evaluación. Los niveles de concreción en la formulación de los objetivos no solo influye en el éxito del programa, sino que también facilita el proceso evaluativo y lo convierte, por su correcta planificación, en estratégico (Besson, 2008). En nuestro estudio, la mitad de los encuestados afirmó redactar los objetivos de forma genérica, y esta es aproximativa y no estratégica. No obstante, más de la mitad estableció en ellos resultados específicos y plazos de cumplimiento. La baja prevalencia en la especificación cuantitativa en relación con los públicos a los que quieren llegar es un dato negativo (Gregory, 2001), al ser contrario a las características de cuantificación y determinación de a quién, qué, cuándo y a qué parte del programa afectan, según fueron establecidas en la Barcelona Declaration of Measurement Principles (AMEC, 2010).

Cuanto más al inicio del programa se realice la planificación de la evaluación, más maduro y profesional es el ejercicio de las relaciones públicas y 
más estratégico (Besson, 2008). A la vista de los resultados, en nuestro estudio no se cumple este criterio, ya que la mayoría de encuestados $(81.4 \%$, $\mathrm{n}=57$ ) no abordaron la evaluación hasta que estaba concluido el programa. Además, en el momento de concretar los objetivos, no la tuvieron en consideración, y contradijeron a Gregory (1996), quien concibe la evaluación como un proceso continuado, considerado desde el momento de establecer los objetivos. Esta dinámica de trabajo, definida por Dozier (1985) como seudoevaluaciones, puede llevar a mediciones simplistas en las que solo se considera el resultado y no el "efecto", por lo que se les resta credibilidad a los procesos y a los profesionales. El planteamiento de la evaluación al final la limita a la salida del programa, y así concuerda con los estudios de Gregory (2001), Pohl y Vandeventer (2001), Walker (1994) y Watson (1997), entre otros.

Refiriéndonos a los resultados del estudio, la elaboración de informes es unánime entre todos los responsables, y facilita, por un lado, la difusión de los resultados de la evaluación y, por otro, ejerce a modo de herramientas que permiten llevar a cabo reajustes en los programas, realizar planificaciones futuras y tomar decisiones (Weiss, 1991; Stacks, 2010).

Finalmente, estas evidencias apuntan a que los hospitales aún se encuentran en una fase embrionaria de adopción de un modelo de aplicación estratégica de la evaluación de sus funciones. Si bien es verdad que la mayoría de los departamentos de relaciones públicas y comunicación de los hospitales del Sistema Nacional de Salud realizan evaluación de sus acciones, destinan a ello una pequeña parte de sus presupuestos. En segundo lugar, las evaluaciones son utilizadas como herramientas de análisis (opiniones y actitudes de los públicos de interés), para redefinición de acciones y futuras planificaciones, sin vincular estos elementos a criterios de planificación estratégica. En tercer lugar, la rendición de cuentas realizada a través de la redacción de informes se dirige preferentemente a la alta dirección, aunque se desvincula de la demostración de aportación de valor de la disciplina de las relaciones públicas ante las "coaliciones dominantes". Por último, el momento tardío en el que se plantea la evaluación en el proceso de la planificación del programa puede dar lugar a seudoevaluaciones, 
en las que se miden los resultados en sí mismos, desalineados de los objetivos del programa.

\section{Referencias}

AMEC, International Association for the Measurement and Evaluation of Communications (2010). Barcelona Declaration of Measurement Principles. Londres: AMEC. Recuperado de http://amecorg.com/ wp-content/uploads/2012/06/Barcelona_Principles.pdf

Díaz, H. A. y Cuesta Cambra, U. (2014). La comunicación de las organizaciones no lucrativas del ámbito de la salud en España: aproximaciones a una matriz de análisis. Historia y Comunicación Social, 2(1), 209-219.

Besson, N. A. (2008). Strategische PR-evaluation: Erfassung, BewertungUndKontrolle von Öffentlichkeitsarbeit. Wiesbaden: VS Verlag.

Bissland, J. H. (1990). Accountability gap: Evaluation practices show improvement. Public Relations Review, 16(2), 25-35.

Bosilkovski, C. y Lee, M. J. (2013). Public relations roles and perceived power in US hospitals. Journal of Communication Management, 17(3), 198-215.

Busto Salinas, L. (2016). ¿Cómo analizar las características de los departamentos de comunicación en los hospitales y sus consecuencias en el ciudadano? Ejemplo metodológico. Revista de Comunicación de la SEECI, 39, 105-126.

Costa Sánchez, C. (2012). El gabinete de comunicación del hospital: propuesta teórica y acercamiento a la realidad de los departamentos de comunicación de los hospitales públicos de Galicia. Doxa Comunicación: revista interdisciplinar de estudios de comunicación y ciencias sociales, 14, 175-197. 
Cutlip, S. M., Center, A. H. y Broom, G. M. (1985). Effective public relations. Englewood Cliffs, NJ: Prentice-Hall.

Dozier, D. M. (agosto, 1981). The diffusion of evaluation methods among public relations practitioners. Ponencia presentada en Annual Meeting of the Association for Education in Journalism, East Lansing, MI.

Dozier, D. M. (1984). Program evaluation and the roles of practitioners. Public Relations Review, 10(2), 13-21.

Dozier, D. M. (1985). Planning and evaluation in PR practice. Public Relations Review, 11(2), 17-25.

Dozier, D. M. (1990). The innovation of research in public relations practice: Review of a program of studies. Journal of Public Relations Research, 2(1-4), 3-28.

Fairchild, M. (2002). Evaluation: An opportunity to raise the standing of PR. Journal of Communication Management, 6(4), 305-307.

Gregory, A. (2000). Planning and managing public relations campaigns. Londres: Kogan Page Publishers.

Gregory, A. (2001). Public relations and evaluation: Does the reality match the rhetoric? Journal of Marketing Communications, 7(3), 171-189.

Grunig, J. E. (2013). Excellence in public relations and communication management. Nueva York, Londres: Routledge.

Grunig, J. E. y Hunt, T. (1984). Managing public relations. Nueva York: Holt, Rinehart and Winsto.

Hogan, B., Hershey, L. y Ritchey, S. (2008). A Case study using a patient satisfaction survey to improve the delivery and effectiveness of drug 
addiction treatment services: Marketing implications and organizational impact. Health Marketing Quarterly, 24(1-2), 93-106.

Huertas, R. (2009). Los sistemas de evaluación en las campañas de relaciones públicas en España. En Relaciones públicas en la gestión de la comunicación interna (pp. 149-157). Sevilla: Asociación de Investigadores en Relaciones Públicas.

Jeffrey, L. y Brunton, M. (2012). Professional identity: How communication management practitioners identify with their industry. Public Relations Review, 38(1), 156-158.

Kvale, S.(1996). Interviews: An introduction to qualitative research interviewing. Thousand Oaks, CA: Sage.

Lindenmann, W. K. (1990). Research, evaluaton and measurement: A national perspective. Public Relations Review, 16(2), 3-16.

Lindenmann, W. K. (1993). An 'effectiveness yardstick' to measure public relations suc. Public Relations Quarterly, 38(1), 7.

Lindenmann, W. (2002). Guidelines for measuring the effectiveness of PR programs and activities. Miami, FL: Institute for Public Relations.

Macnamara, J. (1999). Research in public relations: A review of the use of evaluation and formative research. Asia Pacific Public Relations Journal, 1(2), 107-133.

Macnamara, J. (2002). Research and evaluation. En C. Tymson, P. Lazar y R. Lazar (eds.), The new australian and new zealand public relations manual (pp. 100-135). Sídney: Tymson Communications.

Matilla, K. y Marca, G. (2012). The accountability gap: The use of preliminary and evaluative research in Spain. Public Relations Review, $38(5), 825-830$. 
Medina Aguerrebere, P. (2012). El valor estratégico de la comunicación interna hospitalaria. Revista de Comunicación y Salud, 2(1), 19-28.

Ministerio de Sanidad y Consumo (2010). Catálogo Nacional de Hospitales. Recuperado de http://www.msc.es/ciudadanos/prestaciones/centrosServiciosSNS/hospitales/

Noble, P. (1999). Towards an inclusive evaluation methodology. Corporate Communications: An International Journal, 4(1), 14-23.

Paine, K. D. (2007). Measuring public relationships: The data-driven communicator's guide to success. Durham, New Hampshire: KDPaine \& Partners.

Pohl, G. y Vandeventer, D. (2001). The workplace, undergraduate education and career preparation. En R. L. Heath (ed.), Handbook of public relations (pp. 357-368). Londres: Sage.

Radford, G. P. y Goldstein, S. Z. (2002). The role of research methods in corporate communication. Corporate Communications: An International Journal, 7(4), 252-256.

Simmons, P. y Watson, T. (2005). Public Relations evaluation in australiapractices and attitudes across sectors and employment status. Asia Pacific Public Relations Journal, 6(2), 1.

Stacks, D. W. (2006). Dictionary of Public Relations Measurement and Research. Miami, FL: Institute for Public Relations.

Stacks, D. W. (2010). Primer of public relations research. Nueva York: The Guilford Press.

Theilmann, R. y Szondi, G. (2009). Public relations research and evaluation. En R. Tench y L. Yeomans (eds.), Exploring public relations (pp. 198-221). Edinburg, UK: Pearson Education. 
Walker, G. (1994). Communicating public relations research. Journal of Public Relations Research, 6(3), 141-161.

Walker, G. (1997). Public relations practitioners' use of research, measurement, and evaluation. Australian Journal of Communication, 24(2), 97.

Watson, T. (julio, 1994). Public relations evaluation: Nationwide survey of practice in The United Kingdom. Ponencia presentada en el International Public Relations Research Symposium, Bled, Slovenia.

Watson, T. (1997). Measuring the success rate: Evaluating the PR process and PR programmes. En P. Kitchen (ed.), Principles and practice of public relations (pp. 283-299). Londres: International Thomson Business Press.

Watson, T. (2008). Public relations research priorities: A delphi study. Journal of Communication Management, 12(2), 104-123.

Watson, T. (2012). The evolution of public relations measurement and evaluation. Public Relations Review, 38(3), 390-398.

Watson, T. y Noble, P. (2007). Evaluating public relations. Londres, Sterling: Kogan Page.

Watson, T. y Simmons, P. (julio, 2004). Public relations evaluation-survey of australian practitioners. Ponencia presentada en el Proceedings of the ANZCA 04 conference, Sydney, Australia.

Watts, R. (2006). What is the role of public relation theory? Journal of Communication Management, 10(1), 103-105.

Weiss, C.H. (1991). Investigación evaluativa: métodos para determinar la eficacia de los programas de acción. México: Trillas.

Wilcox, D. L., Cameron, G. T. y Reber, B. H. (1992). Public relations: Strategies and tactics. Nueva York: HarperCollins. 
Wise, K. (2009). Public relations and health diplomacy. Public Relations Review, 35(2), 127-129.

Wise, K. (2012). Vision and state health department public relations practitioners: Prepared to lead? Public Relations Review, 38(4), 592-599.

Wright, D., Gaunt, R., Leggetter, B., Daniels, M. y Zerfass, A. (2009). Global survey of communications measurement 2009-final report. Londres: Association for Measurement and Evaluation of Communication.

Xavier, R., Mehta, A. y Gregory, A. (2006). Evaluation in use: The practitioner view of effective evaluation. PRism, 4(2), 1.

Xavier, R., Johnston, K., Patel, A., Watson, T. y Simmons, P. (2005). Using evaluation techniques and performance claims to demonstrate public relations impact: An australian perspective. Public Relations Review, 31(3), 417-424. 